\title{
Programa de Atenção Integral à Saúde do Artista de Performance: relato da experiência desenvolvida em um serviço universitário em Minas Gerais*
}

\section{Comprehensive Health Care Program for Performing Artists: account of the experience developed in an University service in Minas Gerais}

\author{
Ronise Costa Lima ${ }^{1}$, Talita Naiara Rossi da Silva ${ }^{2}$, Gisele Beatriz de Oliveira Alves ${ }^{3}$, \\ Rosana Ferreira Sampaio ${ }^{4}$, João Gabriel Marques Fonseca ${ }^{5}$, Leonardo Lobão \\ Lacerda $^{6}$, Tarcísio Márcio Magalhães Pinheiro ${ }^{7}$
}

http://dx.doi.org/10.11606/issn.2238-6149.v27i2p221-227

\begin{abstract}
Lima RC, Silva TNR, Alves GBO, Sampaio RF, Fonseca JGM, Lacerda LL, Pinheiro TMM. Programa de Atenção Integral à Saúde do Artista de Performance: relato da experiência desenvolvida em um serviço universitário em Minas Gerais. Rev Ter Ocup Univ São Paulo. 2016 maio-ago.;27(2):221-7.
\end{abstract}

\begin{abstract}
RESUMO: Trata-se de um relato de experiência cujo objetivo foi apresentar o Programa de Atenção Integral a Saúde do Artista de Performance do Serviço Especializado em Saúde do Trabalhador do Hospital das Clínicas da Universidade Federal de Minas Gerais e relatar as ações desenvolvidas pela equipe interdisciplinar. As ações compreendem avaliação clínico-ocupacional, incluindo análise da atividade com o instrumento musical; visitas técnicas aos locais de trabalho, assistência individual ou atividades em grupo e, ações educativas e preventivas, como a elaboração de um folheto informativo e workshops sobre a saúde do músico. De dezembro de 2009 a maio de 2015 foram atendidos 122 músicos, sendo a maioria instrumentistas, do sexo masculino, com média de idade de 32 anos. As reavaliações e os relatos dos trabalhadores assistidos demonstraram que os objetivos do programa vêm sendo alcançados, principalmente no que diz respeito ao autogerenciamento das estratégias de enfrentamento dos riscos de adoecimento no trabalho. Para dar sustentação científica aos resultados, foi elaborado um protocolo sistematizando as atividades em grupo, que se encontra em fase de avaliação. Espera-se que a experiência do SEST com relação a saúde do músico possa auxiliar outros profissionais e serviços a se organizar frente as demandas deste coletivo.
\end{abstract}

DESCRITORES: Saúde do trabalhador; Música; Doenças ocupacionais/prevenção \& controle; Assistência integral à saúde; Exposição ocupacional/efeitos adversos; Serviços de saúde do trabalhador.
Lima RC, Silva TNR, Alves GBO, Sampaio RF, Fonseca JGM, Lacerda LL, Pinheiro TMM. Comprehensive Health Care Program for Performing Artists: account of the experience developed in an University service in Minas Gerais. Rev Ter Ocup Univ São Paulo. 2016 May-Aug.;27(2):221-7.

\begin{abstract}
This is an experience report whose aim was to present the Comprehensive Health Care Program for Performing Artists of the Specialized Service in Occupational Health at the Hospital das Clínicas of the Federal University of Minas Gerais and report the actions developed by the interdisciplinary team. Actions comprise occupational clinical evaluation, including analysis of the activity with musical instruments; technical visits to workplaces, individual assistance or group activities and, educational and preventive actions, such as the preparation of a leaflet and workshops about musician's health. From December 2009 to May 2015, 122 musicians were assisted, most of them instrumentalists, male, with a mean age of 32 years. Reevaluations and reports of workers assisted showed that program objectives have been met, especially regarding the self-management of coping strategies of illness risks at work. To give scientific support to the results, a protocol systematizing group activities, which is under evaluation, was prepared. It is hoped that the experience of the SEST regarding musician's health can assist other professionals and services to organize themselves to cope with this group's demands.
\end{abstract}

KEYWORDS: Occupational health; Music; Occupational health/control \& prevention; Comprehensive health care; Occupational exposure/adverse effects; Occupational health services.

* Este Programa deu origem ao projeto de pesquisa de doutorado da primeira autora; em andamento na Universidade Federal de Minas Gerais, no Programa de Pós-Graduação em Ciência da Reabilitação do Departamento de Fisioterapia e Departamento de Terapia Ocupacional.

1. Universidade Federal de Minas Gerais. Departamento de Fisioterapia e Departamento de Terapia Ocupacional. E-mail: limaronise@gmail.com

2. Universidade Federal de Minas Gerais. Departamento de Terapia Ocupacional. E-mail: talitarossi@ufmg.br

3. Universidade Federal de Minas Gerais. Departamento de Terapia Ocupacional. E-mail: giseleal@eeffto.ufmg.br

4. Universidade Federal de Minas Gerais. Departamento de Fisioterapia. E-mail: rosana.sampaio@pq.cnpq.br

5. Universidade Federal de Minas Gerais. Faculdade de Medicina. E-mail: joaogabriel@medicina.ufmg.br

6. Universidade Federal de Minas Gerais. Orquestra Sinfônica da Escola de Música da UFMG. E-mail: violeo100@hotmail.com

7. Universidade Federal de Minas Gerais. Faculdade de Medicina da UFMG. E-mail: tmmp@medicina.ufmg.br

Endereço para correspondência: Ronise Costa Lima. Serviço Especializado em Saúde do Trabalhador. Ambulatório Bias Fortes. Alameda Álvaro Celso, 175. $7^{\circ}$ andar, Santa Efigênia. Belo Horizonte, MG. CEP 30150-160. E-mail: limaronise@gmail.com 


\section{INTRODUÇÃO}

$\mathrm{O}$ trabalho do músico no Brasil, em que pese o crescimento deste grupo ocupacional, vem mantendo características que demonstram instabilidade e intermitência, comprovadas pelo reduzido número de profissionais com contrato de trabalho formal e elevado número de autônomos. O setor público, mais especificamente a docência no ensino superior e a vinculação as orquestras têm sido as principais alternativas de trabalho formal para os profissionais da música. No entanto, até mesmo em instituições públicas, é comum a contratação destes trabalhadores como prestadores de serviços temporários, situação que vem ocorrendo desde 1990, decorrente das reestruturações das orquestras por razões políticas, econômicas e artísticas ${ }^{1}$.

A situação de vulnerabilidade dos profissionais da música é bastante singular, se considerarmos que trata-se em sua maioria, de trabalhadores altamente qualificados, com vínculos de trabalho precários, o que ocasiona uma grave perda de direitos relacionados ao trabalho intermediada por ações e políticas do Estado ${ }^{1}$.

Em um contexto social em que prevalece a razão instrumental, observa-se a valorização do homo economicus levando a aceleração do processo produtivo e, em consequência, ao sofrimento do trabalhador ${ }^{2}$. Ambos os aspectos funcionam como fatores de risco para o aparecimento de distúrbios osteomusculares, os quais, quando relacionados à atividade laboral, têm como possíveis determinantes a organização do trabalho e da produção, a concepção de ferramentas e equipamentos e o ambiente físico ${ }^{3}$.

No caso específico da saúde dos músicos, a literatura nessa temática destaca a elevada prevalência de distúrbios musculoesqueléticos, desde a década de 1980, além de alterações no sono e sofrimento associados a situações de estresse e ansiedade ${ }^{4-8}$. Estudos mostram que estes distúrbios já atingiam, nesta década, cerca de 59\% de músicos instrumentistas americanos, em uma amostra composta por 250 participantes, entre estes professores, estudantes, profissionais e artistas amadores. No estudo, a inadequação do mobiliário e da iluminação foi apontada como responsável pelo aumento da tensão muscular e dores no pescoço e nas costas ${ }^{4}$. A ansiedade relacionada à qualidade do desempenho mostrou associação significativa com às queixas musculoesqueléticas nessa população9,10.

Somado a condições específicas da atividade musical, a busca por melhor remuneração financeira leva o músico a ter uma maior carga horária de trabalho e em diferentes ambientes, o que aumenta e diversifica a exposição ao risco. Além de escassas, medidas preventivas e de proteção à saúde só são adotadas, na maioria das vezes, após o adoecimento. A elaboração de estratégias nem sempre adequadas para o enfrentamento dos riscos contribuem para a manutenção dos sintomas ou surgimento de outros agravos à saúde ${ }^{11}$.

A partir de uma revisão de estudos publicados entre 1980 e 1996, Zaza ${ }^{12}$ encontrou que a prevalência de distúrbios musculoesqueléticos relacionados à prática musical variou entre $39 \%$ e $87 \%$ em músicos adultos e $34 \%$ e $62 \%$ entre estudantes de música de nível secundário. Apesar da variabilidade, vale destacar a expressiva prevalência encontrada.

Estudo realizado na Austrália em 2012 salientou que $84 \%$ dos músicos, em uma amostra com 377 participantes, relataram experiências de dor ou lesões que interferiram no desempenho musical e na sua participação na orquestra, em algum momento da carreira. A metade dos participantes relatou sintomas álgicos ou lesão durante a realização da pesquisa e 39\% associaram suas queixas ao trabalho. A sobrecarga da prática e a alta exigência da performance musical foi relacionada ao aparecimento de lesões e dores por $75 \%$ dos músicos ${ }^{13}$.

Estudos que avaliaram a frequência de adoecimento entre músicos eruditos e populares mostrou que ambos são acometidos por transtornos musculoesqueléticos. Arnason et al. ${ }^{14}$ encontraram uma prevalência mais elevada entre estudantes de música erudita quando comparados com estudantes de música popular. Os autores argumentaram que esta diferença pode ser explicada pelas demandas específicas dos instrumentos e pela postura adotada ao tocar.

Quando a comparação ocorreu entre músicos da mesma categoria (músicos de orquestra), apesar da prevalência mais alta entre instrumentistas de sopro e de cordas, a frequência de queixas/sintomas não apresentou diferença significativa entre os naipes, ou seja, cordas, sopro e madeiras 5 .

Estudo realizado com 243 músicos de seis orquestras sinfônicas britânicas identificou que $86 \%$ dos profissionais apresentaram dor musculoesquelética, no ano que antecedeu o estudo, e entre esses, $41 \%$ reportaram dor incapacitante ${ }^{15}$.

No Brasil, estudos com músicos também alertaram para o adoecimento dos instrumentistas de orquestras no país. A pesquisa desenvolvida por Andrade e Fonseca ${ }^{16}$ envolveu 419 músicos de diferentes estados e constatou a ocorrência de desconforto físico em $88 \%$ dos participantes. Os sintomas predominantes foram dor e cansaço, referidos por $65 \% \mathrm{e}$ $45 \%$ dos músicos, respectivamente, seguidos por dormência e contração muscular involuntária. O desconforto físico foi 
relacionado à prática musical por $67 \%$ dos participantes, resultando na interrupção da atividade em $30 \%$ dos casos ${ }^{16}$.

Um estudo realizado com 241 músicos das seis maiores Orquestras Sinfônicas da cidade de São Paulo apontou que $63 \%$ dos participantes sofriam com distúrbios do sono e $88 \%$ com estresse psicológico. A frequência de dor foi elevada, acometendo $65 \%$, sendo que a metade relatou impacto moderado ou severo da dor no desempenho artístico. Ademais, 55\% reportaram dor em três ou mais partes do corpo e cerca de $73 \%$ sintomatologia por período superior a um ano ${ }^{17}$.

No referido estudo, os fatores citados como desencadeadores de dor foram sobrecarga de trabalho (32\%), postura inadequada $(27 \%)$ e estresse emocional (26\%). Para estes músicos, os fatores que mais se associaram ao estresse foram a elevada exigência de si mesmo $(73 \%)$, medo de falhar $(50 \%)$, ansiedade $(50 \%)$ e tensão (45\%). Todos estes fatores apresentaram correlação significativa com o aparecimento da dor na amostra analisada. Além disso, os músicos relataram vivenciar dificuldades interpessoais e preocupações com o ambiente de trabalho e ainda, falta de motivação e de autonomia para a realização das tarefas ${ }^{17}$.

Mais da metade dos 33 violinistas das orquestras de Belo Horizonte (51\%) relataram ter problemas de saúde sendo as queixas principais dor, fadiga muscular, câimbra ou formigamento. As partes do corpo mais atingidas foram coluna cervical e lombar e membros superiores. Apenas $35 \%$ dos violinistas adotavam medidas preventivas como alongamentos ${ }^{18}$.

Oliveira e Vezzá $a^{6}$ encontraram queixas de dores musculoesqueléticas em $93 \%$ dos 69 músicos de uma orquestra da região do $\mathrm{ABCD}$ Paulista e $84 \%$ da amostra associaram estas queixas à atividade musical. As regiões mais afetadas foram pescoço, punho, mãos, dedos e coluna lombar.

Apesar da alta prevalência de queixas e sintomas que sugerem o adoecimento destes profissionais, Kaneko et al. ${ }^{17}$ ressaltaram que $73 \%$ dos músicos negaram ter problemas de saúde, demonstrando naturalização da dor. Esse fato dificulta o reconhecimento da necessidade e importância da adoção de medidas preventivas e da busca por tratamento.

Os estudos citados evidenciaram elevada prevalência de distúrbios musculoesqueléticos e indicaram possíveis correlações destes com aspectos do trabalho entre músicos profissionais e estudantes, em diferentes países. Frente a tal realidade, observa-se a importância de ações visando o diagnóstico, tratamento, educação em saúde e prevenção do adoecimento entre estes trabalhadores na expectativa de promover transformações nas relações entre trabalho e saúde neste campo. Nesta perspectiva, o presente trabalho consiste de um relato de experiência que tem como objetivo apresentar o Programa de Atenção Integral a Saúde do Artista de Performance do Serviço Especializado em Saúde do Trabalhador do Hospital das Clínicas da Universidade Federal de Minas Gerais (SEST/HC/UFMG) e as ações desenvolvidas pela equipe interdisciplinar.

\section{PROGRAMA DE ATENÇÃO INTEGRALA SAÚDE DO ARTISTA DE PERFORMANCE}

Em função do alto índice de adoecimento entre músicos demonstrado pela literatura e confirmado pela clínica, o SEST-HC/UFMG implantou o Programa de Atenção Integral à Saúde do Artista de Performance, via Sistema Único de Saúde (SUS), em 2009.

O programa tem como objetivo desenvolver ações de saúde para trabalhadores do meio artístico, tais como dançarinos, atores e músicos, englobando atividades assistenciais individuais e coletivas, prevenção de doenças, promoção e vigilância em saúde do trabalhador. Até o momento, as ações e atividades desenvolvidas no programa foram direcionadas à saúde do profissional da música. Tais ações compreendem avaliações do indivíduo e do desempenho da atividade musical no ambiente de trabalho. O olhar dos diferentes profissionais que compõem a equipe, voltados para o indivíduo e para o seu trabalho, ancoram as decisões de quais ações serão implementadas em cada caso.

Por se tratar de consulta pré-agendada, para auxiliar na análise da atividade e no levantamento de possíveis associações entre queixas e modo de operar o instrumento, é solicitado ao músico, quando possível, levar o seu instrumento no dia da avaliação.

$\mathrm{O}$ acesso ao programa ocorre por livre demanda e, em alguns casos, os músicos são encaminhados por colegas já assistidos pelo SEST, professores, profissionais que atuam em um serviço privado especializado em Saúde do Músico (Núcleo de Atenção Integral à Saúde do Músico - ExerSer) e, atualmente está disponível um folheto para divulgar as atividades oferecidas.

\section{Como surgiu a proposta do Programa de Atenção Integral à Saúde do Artista de Performance?}

Em 1999 o ExerSer iniciou suas atividades na cidade de Belo Horizonte, Minas Gerais, com um projeto no qual o músico era assistido por uma equipe interdisciplinar 
composta por um médico que também é músico, uma fisioterapeuta e duas terapeutas ocupacionais.

$\mathrm{O}$ foco de atuação da equipe naquele momento estava voltado para a assistência, mas os profissionais sempre procuraram compreender as questões relacionadas ao trabalho do músico. Nesse sentido, entre 2005 e 2007, foi realizada uma pesquisa objetivando identificar a percepção do músico de orquestra sobre a relação saúdedoença-trabalho ${ }^{19}$.

Os dados encontrados neste estudo, somados a informação disponível na literatura mundial apontaram para a necessidade de criar um programa de atenção integral à saúde destes trabalhadores via SUS e o local escolhido foi o SEST-HC/UFMG. Este serviço foi criado em 1983 e desde então desenvolve ações voltadas para a assistência, a formação de recursos humanos e a pesquisa, atendendo trabalhadores com queixas relacionadas ao trabalho. A expertise dos profissionais, a estrutura e organização do SEST favoreceram a implementação da proposta de cuidado a saúde dos músicos.

\section{Como funciona o programa?}

Como citado anteriormente todos os músicos que acessam o programa são avaliados de forma criteriosa por uma equipe interdisciplinar. Para identificar as queixas e o nexo ocupacional é realizada uma avaliação inicial com a participação de médicos (clínico, do trabalho e residentes), terapeutas ocupacionais, estagiários de terapia ocupacional, psicólogos, fonoaudiólogo e músico. Esta avaliação inclui levantamento de informações sobre o modo operatório e os fatores relacionados ao ambiente e organização do trabalho do músico. Durante a prática com o instrumento são observados o modo de utilização do corpo (ajustes posturais) e a presença ou não de tensão muscular contínua (contração isométrica/sustentação do instrumento ou força excessiva para a realização da tarefa).

Apesar desta avaliação ainda estar focada principalmente no trabalhador e algumas vezes ocorrer em atividade simulada, ou seja, o músico leva seu instrumento paraoSEST,tal procedimento temproduzidodados relevantes que auxiliam na compreensão de possíveis associações da atividade musical com as queixas apresentadas. No entanto, sempre que possível, a equipe realiza visitas técnicas em diferentes locais de estudo ou trabalho destes profissionais, como orquestras e escolas de música.

Complementando a avaliação com o instrumento musical e a anamnese, é realizado um exame físico que consta de testes padronizados que incluem testes provocativos para tendinites, tenossinovites e síndromes compressivas $^{3,20}$, testes de sensibilidade, força muscular e amplitudearticular ${ }^{21,22}$ eainda, administradoumquestionário para avaliar a intensidade da dor e sua interferência na qualidade de vida (Brief Pain Inventory) ${ }^{23}$. $\mathrm{Na}$ anamnese, utilizando um roteiro elaborado especificamente para o programa, são coletadas informações que permitem traçar o perfil clínico-ocupacional dos participantes. Durante as avaliações, mediante autorização do músico, há o registro de imagem (fotografia e filmagem) para acompanhamento dos casos e reavaliações.

Após a discussão dos resultados da avaliação, a equipe decide sobre a modalidade de acompanhamento de cada músico, que compreende atividades em grupo (grupo de autogerenciamento da saúde) e assistência individual quando necessário.

A assistência individual é indicada nos casos em que os sintomas e queixas já comprometem a atividade laboral e necessitam de intervenções específicas. De acordo com a demanda, o tratamento fica a cargo do terapeuta ocupacional, psicólogo, fonoaudiólogo ou o trabalhador é encaminhado para outro profissional fora da equipe.

As atividades em grupo são realizadas pela terapia ocupacional com o objetivo de auxiliar no autogerenciamento da saúde e sua relação com o trabalho, por meio do Programa de Autogerenciamento da Saúde do Músico. Este programa busca contribuir para o desenvolvimento da percepção do músico sobre os riscos de adoecimento relacionados ao trabalho e a elaboração de estratégias de enfrentamento.

A proposta do Programa de Autogerenciamento da Saúde do Músico inclui tratamento sintomático, técnicas de adequação postural na realização das atividades, readequação da técnica de performance e criação de ambiente que propicie a elaboração de estratégias de autogerenciamento para o enfrentamento dos riscos de adoecimento causados pela atividade laboral. Para a readequação da técnica há assessoria de músicos profissionais, em geral ligados às escolas de música da Universidade Estadual de Minas Gerais (UEMG) e da UFMG.

A prática da assistência em grupo foi incorporada ao Programa considerando que as demandas trazidas pelos músicos se assemelhavam. Ao longo de dois anos o Grupo de Autogerenciamento foi aprimorado com a participação dos músicos e passou a ser utilizado um roteiro estruturado com os temas e atividades a serem realizadas em cada encontro. Para dar sustentação científica aos resultados do trabalho foi desenvolvido um protocolo que sistematiza evidências e as práticas em grupo, que se encontra em fase de avaliação.

No referido protocolo são propostos oito encontros que têm temas específicos e ocorrem na seguinte sequência: 
1) Consciência do padrão respiratório, relaxamento fisiológico e muscular progressivo; 2) Automassagem e percepção das tensões do corpo; 3) Exercícios ativos suaves para aquecimento; 4) Alongamentos e percepção da flexibilidade do corpo; 5 e 6) Consciência postural em atividade e percepção das tensões corporais durante a atividade; 7) Ansiedade de performance e realização de exercícios de sensopercepção, recurso corporal e Tapas Accupressure Technique (TAT); 8) O processo de trabalho coletivo, questões previdenciárias e elaboração das estratégias para antes e depois da prática musical.

À medida que o trabalhador consegue perceber os riscos e conhecer as estratégias de enfrentamento dos mesmos, é possível que venha adquirir maior domínio sobre a dicotomia saúde-doença e passe a ter mais subsídios para prevenir-se do adoecimento.

Uma estratégia para motivar os participantes, aumentar a adesão às práticas aprendidas e o uso contínuo após a alta do serviço, foi a confecção de uma cartilha de automassagem, aquecimento e alongamento.

É importante ressaltar que, nesta proposta, muitos trabalhadores têm realizado o tratamento e aprendido as estratégias de autogerenciamento sem a necessidade de se afastar das suas atividades laborativas. Como grande parte dos músicos atendidos atua também como professor, tem sido possível difundir entre os alunos os conhecimentos adquiridos durante o tratamento e, dessa forma, os participantes dos grupos têm atuado como multiplicadores das ações do SEST.

Além da assistência individual e das atividades em grupo, estão sendo realizadas ações coletivas. Estas ações incluem workshops em locais de estudo e trabalho de músicos e visam a conscientização sobre o trabalho e a sua relação com a saúde e a doença.

Nesse sentido, foi realizado em 2015 um workshop sobre Saúde do Músico na Orquestra da Escola de Música da UFMG, visando a educação em saúde e prevenção do adoecimento relacionado ao trabalho do músico. Esta ação ocorreu em cinco encontros e foram envolvidos estudantes da graduação em música e músicos profissionais. No mesmo ano, por solicitação dos gestores da Orquestra Filarmônica de Minas Gerais, um ciclo de palestras foi realizado, em parceria com o (ExerSer).

\section{Como o Programa tem colaborado com o ensino, a pesquisa e a extensão?}

O Programa colabora com a formação de residentes em Medicina do Trabalho e estudantes de graduação em Terapia Ocupacional matriculados na disciplina Clínica em
Terapia Ocupacional na Saúde do Adulto e do Idoso. Esta disciplina caracteriza-se pelo ensino supervisionado em serviços dos campos da saúde e proteção social, visando a vivência dos discentes em situações reais de prática.

Pesquisas estão sendo desenvolvidas a partir das experiências no Programa para avaliar a efetividade do protocolo do grupo (Programa de Pós-graduação em Ciências da Reabilitação da UFMG) e compreender as relações entre trabalho e saúde do músico (Programa de Mestrado em Psicologia/Relações Psicossociais da Pontifícia Universidade Católica, PUC-Minas).

Atividades de extensão no Programa foram iniciadas em 2015 por meio da parceria com o projeto Ações Interdisciplinares em Saúde do Trabalhador do Departamento de Terapia Ocupacional da UFMG. Esta parceria contribuiu com a elaboração de um folheto informativo e colaborou na articulação junto à Orquestra da Escola de Música da UFMG e na realização do workshop mencionado anteriormente. Tais ações serão continuadas em 2016.

\section{Quais os resultados até o momento?}

Em cinco anos de funcionamento, foi atendido no Programa um total de 122 músicos, sendo $72 \%$ do sexo masculino e $28 \%$ do feminino. A média de idade foi de 32 anos. Há grande variabilidade na idade dos participantes, apresentando uma amplitude de 62 anos, com idade mínima de 17 e máxima de 79 anos. Dentre estes, 25\% têm entre 17 e 23 anos (Q1=23), 50\% têm até 27 anos $(\mathrm{Q} 2=27)$ e $25 \%$ tem até 42 anos $(\mathrm{Q} 3=42)$, sinalizando que a maioria dos músicos assistidos até o momento é de jovens trabalhadores.

Dois dos músicos atendidos se dedicavam à regência, $11(9 \%)$ ao canto e os demais (89\%) eram instrumentistas, incluindo os que tocavam mais de um instrumento musical. Os instrumentos mais frequentes foram violão (25), violino (11), percussão (10), contrabaixo acústico (9), piano (8), guitarra (8) e violoncelo (6).

Os principais sintomas apresentados pelos músicos foram dor espontânea ou na atividade (89\%), fadiga, cansaço ou sensação de peso (30\%), movimento involuntário (11\%) e rigidez $(7 \%)$, salientando que a maioria apresentava mais de um sintoma. Estes dados relacionados aos músicos atendidos no SEST coincidem com a prevalência apresentada na literatura ${ }^{5,6,12,13,15,16}$.

No período analisado, foi possível verificar que $75 \%$ dos músicos apresentaram algum distúrbio postural primário e/ou inadequação na técnica de performance do instrumento, levando a sintomas como dor, rigidez e fadiga muscular; $21 \%$ tinham diagnóstico de tendinites 
ou tenossinovites ou outras doenças dos sistemas musculoesqueléticos; $10 \%$ receberam diagnóstico de distonia focal e $2 \%$ de fibromialgia. Os distúrbios posturais mais frequentes foram a projeção anterior da cabeça, retração da musculatura cervical, alteração no grau de cifose torácica e lordose lombar e desnivelamentos (básculas) nas cinturas escapular e pélvica.

No workshop realizado com estudantes e técnicos da Orquestra da Escola de Música da UFMG participaram 48 músicos. Entre estes, $88 \%$ referiram ter sentido algum desconforto físico nos últimos 12 meses que antecederam a participação, principalmente, dor, tensão muscular, fadiga e contração muscular involuntária, e $75 \%$ associaram este desconforto à prática musical.

\section{CONSIDERAÇÕES FINAIS E PERSPECTIVAS FUTURAS}

Os fatores de risco para o surgimento de distúrbios osteomusculares têm como determinantes a organização do trabalho e da produção, a concepção de ferramentas e equipamentos, o ambiente físico, a inadequação do mobiliário, a sobrecarga de trabalho, postura inadequada e estresse emocional, gerados pela elevada exigência de si mesmo, pelo medo de falhar e pelas dificuldades interpessoais no trabalho $0^{3,4,11,13,17}$. Para reduzir os fatores de risco de modo mais efetivo seria necessário atuar em todos estes determinantes de forma integrada.

No entanto, apesar da compreensão de que o trabalhador não tem responsabilidade total por sua condição de saúde no trabalho, é possível que auxiliálo na percepção dos fatores de risco e na elaboração de estratégias para o autogerenciamento da sua saúde na relação com o trabalho, viabilizando a aquisição de habilidades em identificar o que pode ser modificado e como agir na impossibilidade da mudança imediata, para minimizar alguns destes determinantes.

Todavia, apesar de o Programa de Autogerenciamento da Saúde do Músico ter suas atividades direcionadas para o sujeito trabalhador, o Programa de Atenção Integral a Saúde do Artista de Performance, do qual ele faz parte, vem consolidando parceria com coordenadores de orquestras e maestros, professores e instituições de ensino da música com o propósito de atuar prevenção, promoção da saúde destes trabalhadores e na compreensão das situações de trabalho que favorecem o adoecimento.

Além das palestras e workshops realizados em Belo Horizonte e outras cidades mineiras, outras apresentando a proposta do Programa de Autogerenciamento da Saúde do Músico também foram realizadas a convite de alguns sindicatos dos músicos como os dos estados do Rio de Janeiro e Amazonas.

As reavaliações e os relatos demonstram que a proposta do Programa de Atenção Integral a Saúde do Músico vem sendo alcançada, principalmente no que diz respeito ao autogerenciamento das estratégias de enfrentamento dos riscos de adoecimento pelo processo de trabalho.

Espera-se que as pesquisas em andamento possam contribuir para a evolução do Programa, incentivar novos estudos, fortalecer as ações implementadas e, somadas às ações coletivas iniciadas em 2015 e as parcerias que vem sendo firmadas, construir ações para compreender e intervir no trabalho dos músicos.

Agradecimentos: A toda a equipe do Serviço Especializado em Saúde do Trabalhador do Hospital das Clínicas da Universidade Federal de Minas Gerais (SEST/HC/UFMG). A Prefeitura Municipal de Betim/MG por ter disponibilizado a primeira autora deste artigo para implantação do Programa de Atenção Integral à Saúde do Músico no SEST/HC/UFMG.

\section{REFERÊNCIAS}

1. Segnini L. O que permanece quando tudo muda? Precariedade e vulnerabilidade do trabalho na perspectiva sociológica. Cad CRH. 2011;24(1):71-88. Disponível em: http://www.scielo.br/pdf/ccrh/v24nspe1/a06v24nspe1.pdf.
2. Uchida S. Organização do trabalho: vivências de prazer e sofrimento. In: Mendes AM, LIMA SCC, Facas EP, organizadores. Diálogos em psicodinâmica do trabalho. Brasília: Paralelo 15; 2007. p.105-18. 
3. Assunção AA, Vilela LVO. Lesões por esforços repetitivos: guia para profissionais de saúde. Piracicaba. São Paulo: Centro de Referência em Saúde do Trabalhador/CEREST; 2009. Disponível em: http://bvsms.saude.gov.br/bvs/ publicacoes/livro_ler_guia_profissional_1.pdf.

4. Caldron PH, Calabrese LH, Clough JD, Lederman RJ, Williams G, Leatherman J. A survey of musculoskeletal problems encountered in high-level musicians. Med Probl Perform Art. 1986;1(4):136-9. Disponível em: https://www.sciandmed.com/mppa/journalviewer. aspx ?issue $=1150 \&$ article $=1500 \&$ action $=1$.

5. Trelha CS, Carvalho RP, Franco SS, Nakaosk Ti, BrozaTP, Fábio TL, Abelha TZ. Arte e saúde: frequência de sintomas musculoesqueléticos em músicos de orquestras sinfônicas da Universidade Estadual de Londrina. Semina Ciên Biol Saúde (Londrina). 2004;25:65-72. DOI: http://dx.doi. org/10.5433/1679-0367.2004v25n1p65.

6. Oliveira CFC, Vezzá FMG. A saúde dos músicos: dor na prática profissional de músicos de orquestra no $\mathrm{ABCD}$ paulista. Rev Bras Saúde Ocup. 2010;35(121):33-40. DOI: http://dx.doi.org/10.1590/S0303-76572010000100005.

7. Paarup HM, Baelum J, Holm JW, Mannich C, Wedderkopp N. Prevalence and consequences of musculoskeletal symtoms in symphony orquestra musicians vary by gender: a cross-sectional study. BMC Muscoleskeletal Disord. 2011;12:223. DOI: 10.1186/1471-2474-12-223.

8. Silva AG, Lã FM, Afreixo V. Pain prevalence in instrumental musicians: a systematic review. Med Probl Perform Art. 2015;30(1):8-19. Available from: https://www. researchgate.net/publication/273147264_Pain_Prevalence_ in_Instrumental_Musicians_A_Systematic_Review.

9. Scheneider E, Chesky K. Social support and performance anxiety of college music students. Med Probl Perform Art. 2011;26(3):157-63. Available from: https://www. researchgate.net/publication/51706665_Social_Support_ and_Performance_Anxiety_of_College_Music_Students.

10. Van Fenema E, Julsing JE, Carlier IV, van Noorden MS, Giltay EJ, van Wee NJ, Zitman FG. Musicians seeking psychiatric help: a preliminary study of psychiatric characteristics. Med Probl Perform Art. 2013;28(1):918. Available from: https://www.researchgate.net/ publication/235796966_Musicians_seeking_psychiatric_ help_A_preliminary_study_of_psychiatric_characteristics.

11. Lima RC, Pinheiro TM, Dias EC, de Andrade EQ. Development and prevention of work related disorders in a sample of Brazilian violinists. Work. 2015;51:273-80. DOI: 10.3233/WOR-141904.

12. Zaza C. Playing-related musculoskeletal disorders in musicians: a systematic review of incidence and prevalence.
CMAJ. 1998;158(8):1019-25. Available from: http://www. cmaj.ca/content/158/8/1019.full.pdf.

13. Ackermann B, Driscoll T, Kenny DT. Musculoskeletal pain and injury in professional orchestral musicians in Australia. Med Probl Perform Art. 2012;27(4):181-7. Available from: https://www.researchgate.net/publication/233941573 Musculoskeletal_Pain_and_Injury_in_Professional_ Orchestral_Musicians_in_Australia.

14. Arnason, K, Arnason, A, Briem, K. Playing-related musculoskeletal disorders among icelandic music students: differences between students playing classical vs rhythmic music. Med Probl Perform Art. 2014;29(2):74-9.

15. Leaver R, Harris EC, Palmer KT. Musculoskeletal pain in elite professional musicians from British Symphony Orchestras. Occup Med. 2011;61:549-55. DOI: 10.1093/occmed/kqr129.

16. Andrade EQ, Fonseca JGM. Artista-atleta: reflexões sobre a utilização do corpo na performance dos instrumentos de cordas. Per Musi (BH). 2000;2:118-29. Disponível em: http://www. musica.ufmg.br/permusi/port/numeros/02/num02_cap_07.pdf.

17. Kaneko Y, Lianza S, Dawson WJ. Pain as an incapacitating factor in Symphony Orchestra Musicians in São Paulo, Brazil. Med Probl Perform Art. 2005;20(4):168174. Available from: https://www.researchgate.net/ publication/285739318_Pain_as_an_incapacitating_factor_ in_symphony_orchestra_musicians_in_Sao_Paulo_Brazil.

18. Lima RC et al. Processo saúde-doença na avaliação da terapia ocupacional e dos saberes dos violinistas de orquestra de Belo Horizonte. Rev Med Minas Gerais. 2006;16(supl 2):118-22.

19. Lima RC. Distúrbios funcionais neuromusculares relacionados ao trabalho: caracterização clínico-ocupacional e percepção de risco por violinistas de orquestra [Dissertação]. Belo Horizonte, MG: Programa de Pós-Graduação em Saúde Pública, Faculdade de Medicina, Universidade Federal de Minas Gerais; 2007. Disponível em: http://www. bibliotecadigital.ufmg.br/dspace/bitstream/handle/1843/ ECJS-7FVHBD/ronise_costa_lima.pdf?sequence $=1$.

20. Barros Filho TEP. Exame físico em ortopedia. 2a ed. São Paulo: Sarvier; 2005.

21. Ferrigno ISV. Terapia da mão. São Paulo: Editora Santos; 2007.

22. Sociedade Brasileira de Terapia da Mão e do Membro Superior. Recomendações para avaliação do membro superior. São Paulo; jun. 2003.

23. Ferreira-Valente MA, Pais-Ribeiro JL, Jensen MP. Interferência da dor na vida diária: validação de uma versão Portuguesa da Escala de Interferência da dor do Brief Pain Inventory. In: V Congresso Internacional de Saúde, Cultura e Sociedade, Viseu, 10-11 jul. 2009. p.164-81.

Recebido em: 29.10.15

Aceito em: 13.01 .16 